\title{
Corrosion Diagnosis and Simulation of Miniature Grounding Grid
}

\author{
Jianhua Zhang ${ }^{\mathrm{a},}{ }^{*}$, Linjing Zhang ${ }^{\mathrm{b}}$, Zhen Wang ${ }^{\mathrm{c}}$ \\ School of electrical engineering, Zhengzhou University, Zhengzhou, China \\ apetermails@zzu.edu.cn, bzlj_zzu@163.com, cwangzhen_ee@163.com
}

Keywords: Grounding network, corrosion, fault diagnosis equation, simulation.

\begin{abstract}
Grounding grid is an important facility to meet the grounding requirements and ensure the safe operation of power plants and substations. The relative resistance of the grounding network branch is closely related to the degree of corrosion and directly affects its grounding performance. The grounding grid is equivalent to a resistance network. Then, the diagnosis equations can be set up, the measured resistance can be solved and the simulation experiment can be carried out. Finally, the corrosion fault of the grounding network branch is accurately located.
\end{abstract}

\section{Introduction}

Grounding grid refers to a network made up of grounding wires connected by a plurality of grounding bodies, and the utility model has the advantages of reliable grounding and small grounding resistance. In China, the power system grounding grid was usually made of galvanized flat steel or angle steel and buried in the ground. The reason of grounding grid corrosion is that the grounding metal and soil dielectric have electrochemical reaction, which will lead to galvanic corrosion cell easily, especially when grounding grid is arranged in corrosive saline alkali soil, the corrosion of metal material will be very serious. Some investigations show that the corrosion rate of metal in different soil is different, and the maximum corrosion rate can be up to $8.0 \mathrm{~mm}$ per year.

In the past decades, many faults occurred in the electrical system. The grounding grid corrosion can led to higher grounding resistance and may cause accident or damage the equipment. Therefore, it is of great importance to find the ground corrosion fault in time.

\section{Fault diagnosis equation and solution}

\subsection{Fault diagnosis equations}

The resistance of the grounding circuit network metal is $10^{6}$ of magnitude, and the soil resistance is $10^{2}$ of magnitude. So, the influence of the soil can be neglected in the analysis of the circuit, and the grounding circuit is approximately equivalent to a pure resistance network.

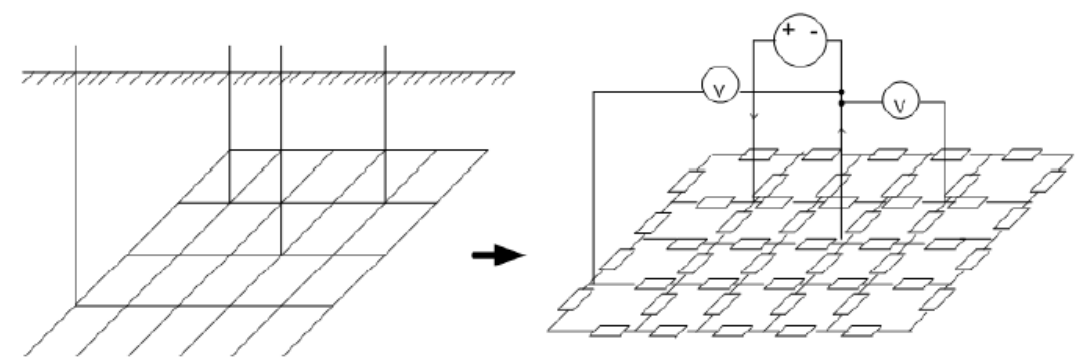

Fig. 1 Equivalent network of a grounding grid

In this way, grounding grid fault diagnosis belongs to analog circuit network diagnosis. The theory of network diagnosis is divided into three types: network analysis, network synthesis and fault diagnosis, see Table 1. 
Table1 The network analysis, synthesis and diagnosis

\begin{tabular}{|c|c|c|c|}
\hline Type & Known condition & Diagnosis factors & $\begin{array}{c}\text { Solution of the } \\
\text { equation }\end{array}$ \\
\hline $\begin{array}{c}\text { network } \\
\text { analysis, }\end{array}$ & Excitation signal and network topology & output response & unique \\
\hline $\begin{array}{c}\text { network } \\
\text { synthesis }\end{array}$ & Excitation signal, output response & network topology & not unique \\
\hline fault diagnosis & $\begin{array}{c}\text { Excitation signal, output response, } \\
\text { network topology }\end{array}$ & $\begin{array}{c}\text { Component } \\
\text { parameter }\end{array}$ & unique requested \\
\hline
\end{tabular}

When the measured current is DC, the grounding grid can be equivalent to the pure resistance grid. Then, grounding grids of data will be measured and with the help of Tellegen's law, the fault diagnosis equations about the relative variation of branch resistance $\Delta R_{i j}$ can be derived .

If the electrical connection fault occurs on the grounding grid, the port resistance of $\mathrm{i}$ and $\mathrm{j}$ terminals of the equivalent electrical network changes from $R_{\mathrm{ij}}$ to $R_{\mathrm{ij}}^{\prime}$ after the occurrence of the fault:

$\Delta R_{i j}=R_{i j}^{\prime}-R_{i j}$

According to Tellegen's law, we can get the following equations:

$b+1$

$\sum_{k=1}^{b+1} U_{k}^{\prime} I_{k}=0$

(2)

$$
\sum_{k=1}^{b+1} U_{k} I_{k}^{\prime}=0
$$

The current source of the equivalent networkin the $\mathrm{b}+1$ branch is $I_{0}$ both before and after the fault, so we can get the following equations:

$$
\begin{aligned}
& I_{\mathrm{b}+1}=I_{b+1}^{\prime}=I_{0} \\
& U_{\mathrm{b}+1}=-R_{i j} I_{0} \\
& U_{b+1}^{\prime}=-R_{i j}^{\prime} I_{0}
\end{aligned}
$$

The most important equation can be derived:

$$
\Delta R_{\mathrm{ij}}=\sum_{k=1}^{b} \Delta R_{k} I_{k}^{\prime} I_{k} / I_{0}^{2}
$$

In the actual diagnosis, the $\mathrm{b}$ order fault diagnosis equations of $\mathrm{b}$ port resistances show as below:

$$
\Delta R_{i j(n)}=\sum_{k=1}^{b} \Delta R_{k} I_{k(n)}^{\prime} I_{k(n)} / I_{0}^{2}, n \in[1, \mathrm{~m}], m<\mathrm{b},
$$

In the quotation, $\mathrm{m}$ is the number of measurable ports, which is smaller than $\mathrm{n}$. $I_{k}$ can be calculated by $R_{k}$ through the known electrical network parameters. But since $I_{k}^{\prime}$ is decided by $R_{k}^{\prime}$, and $R_{k}^{\prime}$ is unknown, the equations can not get the exact solutions directly.

\subsection{Newton iteration method to solve equations}

A schematic diagram of the solution of the Newton iteration is shown in Figure 2.1. The function $\mathrm{f}$ (x) intersects the $\mathrm{X}$ axis at $X^{*}$, so $X^{*}$ is the root of the equation $\mathrm{f}(\mathrm{x})=0$. 


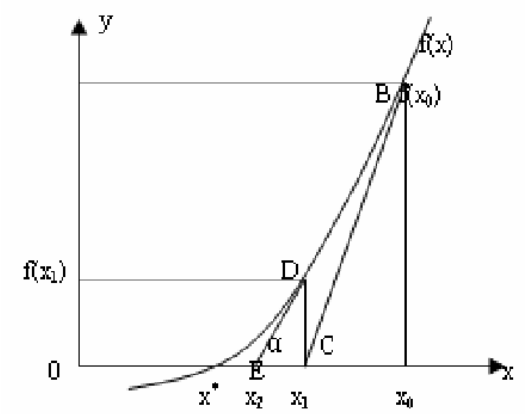

Fig. 2 Newton iteration method

$$
X_{k+1}=X_{k}-H^{-1}\left(X_{k}\right) \nabla f\left(X_{k}\right)
$$

If the initial value is $X_{0}$ is provided, we can use the formula to calculate the value of $X_{1}$, then we can put $X_{1}$ into the formula and get the value of $X_{2}$. In this way, we can get a series of $\left\{X_{n}\right\}$. It can be easily proved that if it is convergent, the value of $X_{n}$ will converge to the stagnation point $X^{*}$.

Similarly, $R_{k}^{t t}=R_{k}+\Delta R_{k}^{(t)}$, when $\mathrm{t} \geq 1$ and the equation $\left|\Delta R_{k}^{(t)}-\Delta R_{k}^{(t-1)}\right|<\varepsilon$ is established, the calculation is right, we can get the solution of $R_{k}^{\prime(t)}, \varepsilon$ is a constant value.

\section{Simulation test of electrical corrosion fault of grounding grid}

When the equations of $\Delta R_{i j}$ were set up, we can use Newton iteration method to solve them, then the fault diagnosis can be made. The steps of the simulation are as follows:

(1) Select the resistance network for the simulation experiment and determine the reference point. Then generate the corresponding correlation matrix A and branch admittance matrix $Y_{b}$ according to the network topology;

Select the accessible port to measure resistance, and calculate the port resistance $R_{i j(s)}$ according to the parameters of the resistance network;

Set fault in the network manually, that is, change the resistance of one branch or several branches in the resistance network, and then calculate the port resistance $R_{i j(s)}^{\prime}$;

According to $\Delta R_{i j}=R_{i j}^{\prime}-R_{i j}$, establish the fault diagnosis equations;

The grounding grid electrical connection fault diagnosis program can be used to calculate the results. The results will be analyzed to make fault diagnosis.

In practice, preliminary diagnosis is made in common fault areas, important electrical facilities and equipment areas. According to the preliminary diagnosis results, the two fault branches are selected for targeted measurement and the second diagnosis needs to be made. When both fault branches are accessible nodes, the port resistances between the two nodes can be measured directly. If not, we can measure the port resistance by selecting the nearest accessible nodes. In this way, the diagnosis can be carried out around the important equipment and the reliability of diagnosis can be ensured. The pseudo fault can be eliminated step by step, so that the real fault can be quickly determined. 


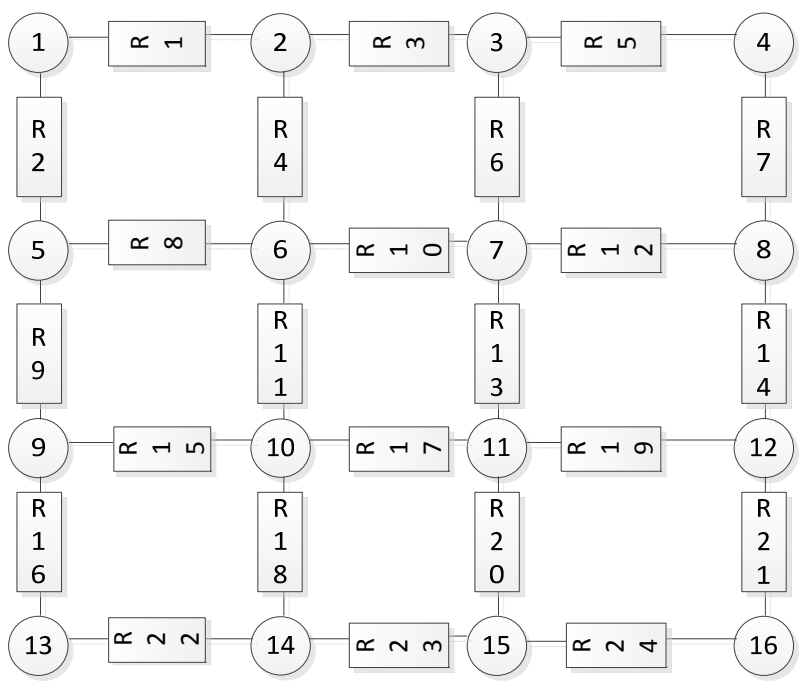

Fig. 3 The diagram of miniature resistance network

Fig. 3 is a topology of a small resistance network with 24 branches and 16 nodes, each of which has a resistance value of $500 \Omega$. If the 17 th branch has an electrical connection failure, the branch resistance is changed to $2500 \Omega$.

Table 2 is the results obtained by measuring selected ports during the diagnosis process and the measured data. Among them, the "measuring node" represents the measurement of the selected network node number. For example, 1-12 means that the measured resistance is a port resistance of nodes 1 and 12. "Diagnostic results" indicate the port resistance values obtained in accordance with this measurement. The branch numbers of the electrical connection failure is obtained by solving the diagnosis equation.

Table 2 The 17 th branch diagnosis

\begin{tabular}{|c|c|c|c|}
\hline Measurement times & Port pair & Diagnostic results & Fault analysis \\
\hline 1 & $1-4,1-16,1-13,1-15$ & 19 & R19 may be a fault \\
\hline 2 & $11-12$ & 17 & R19 is a pseudo fault \\
\hline 3 & $10-11$ & 17 & R17 is the real fault \\
\hline
\end{tabular}

As shown in Figure 4(the abscissa is the branch number and the ordinate is the relative change of the branch electric), the primary diagnosis result is that the branch resistance change of branch 19 is abnormal. According to the principle of selecting adjacent nodes for testing, the fault of the electrical connection fault can be further detected.

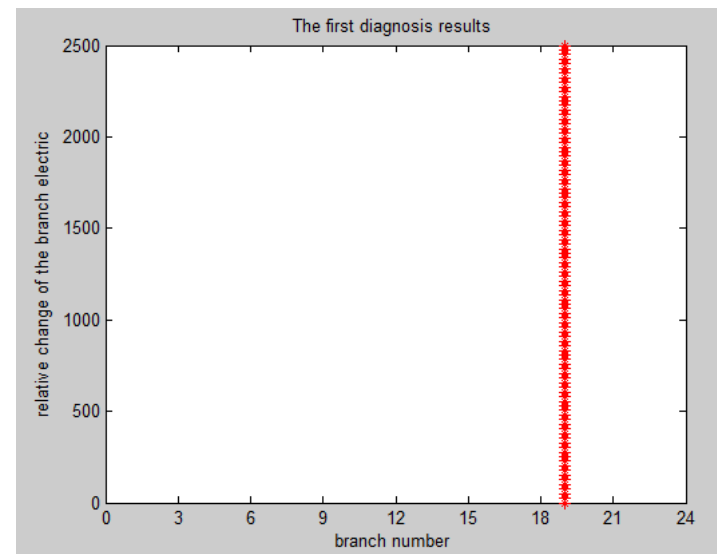

Fig. 4 the $1^{\text {st }}$ diagnosis result

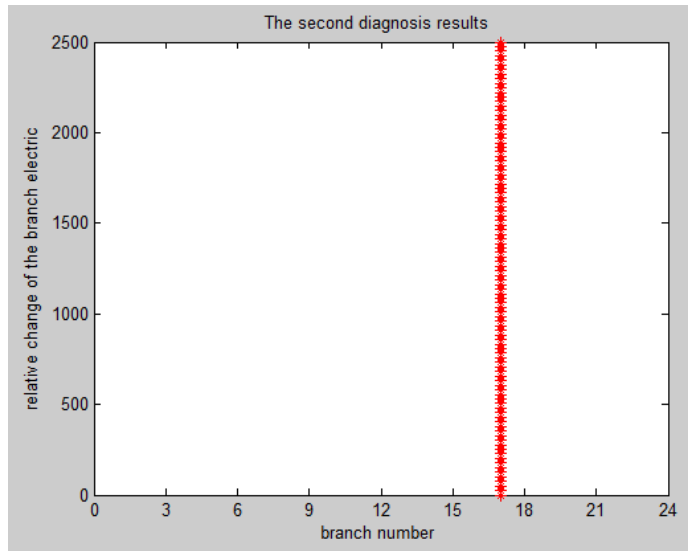

Fig. 5 the $2^{\text {nd }}$ diagnosis result

The port resistance of the 19th branch 11 and 12 is diagnosed two times, and the analyzed result is 17 th branch has fault, as shown in figure 5.The calculation result of two times before and after 19th branch resistance varies greatly, which shows that the fault characteristic is unstable and can be judged to be pseudo fault. 


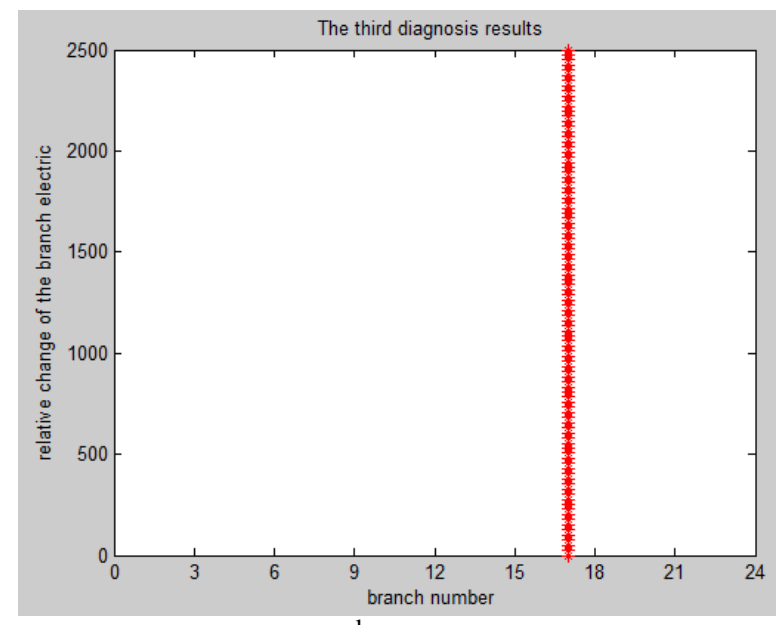

Fig. 6 the $3^{\text {rd }}$ diagnosis result

Similarly, the node 10 and 11 need to be measured again to determine the authenticity of the 17 th branch fault. From the figure 6, the 17th branch is still identified as faulty. The fault characteristic parameter of the branch is stable and can be judged as a real fault, and the diagnosis result agrees with the set fault.

\section{Conclusion}

Grounding grid network is equivalent to the pure resistance network. According to the $\mathrm{M}$ resistance values measured by the accessible nodes and the $\mathrm{N}$ order diagnosis equations, the resistance values can be calculated by Newton iteration method. Then, the simulation experiment is designed to verify it. The correctness and feasibility of the diagnosis principle are verified by analyzing the branch fault of the small grounding network. It is of significance to explore the diagnosis law of electrical corrosion fault of miniature grounding grids

\section{References}

[1] Yuan Tao, Sun Caixin. Research on the Diagnosis Method of the Electrical Connection Faults in Grounding Grid, D. Chongqing University, 2010.

[2] He Jinliang, Zeng Rong, Grounding technology of power system, M. Beijing, Science Press, 2007.

[3] Yu Yafei, Zhan Yuezhang, Lin Zhiwei. Study on Corrosion of grounding grid in 220KV substation of Hubei power grid, J. Hubei electric power, 2007.8, Vol.31 No.4.

[4] Hu Xuewen, Xu Chongwu. Performance test of corrosion protection material for grounding grid, J. High voltage technology, 2002.5, Vol.28.No.5:21-23.

[5] Jun Hu, Rong Zeng, Jinliang He, etc. Novel Method of Corrosion Diagnosis for Grounding Grid[C]. IEEE, 2002.

[6] Chakrabarti S., Chatterjee A. Fault modeling and fault sampling for isolating faults in analog and mixed-signal circuits [C]. ISCAS'99.Proceedings of the 1999 IEEE International Symposium on Circuits and Systems, Jul 1999, Volume: 2, Page(s): 444-447.

[7] Wu Ning, Electrical network analysis and synthesis, M. Beijing, Science Press, 2003. 